\title{
Prevalence and Characteristics of Phenicol-Oxazolidinone Resistance Genes in Enterococcus Faecalis and Enterococcus Faecium Isolated from Food-Producing Animals and Meat in Korea
}

\author{
Eiseul Kim ${ }^{1,+}{ }^{\mathbb{D}}$, So-Won Shin ${ }^{1,+}{ }^{\mathbb{D}}$, Hyo-Sun Kwak ${ }^{1}$, Min-Hyeok Cha ${ }^{2}$, Seung-Min Yang ${ }^{1}$, Yoon-Soo Gwak ${ }^{1}$, \\ Gun-Jo Woo ${ }^{2, *}$ and Hae-Yeong Kim ${ }^{1, *(D)}$
}

1 Institute of Life Sciences \& Resources and Department of Food Science and Biotechnology, Kyung Hee University, Yongin 17104, Korea; eskim89@khu.ac.kr (E.K.); volume1@khu.ac.kr (S.-W.S.); hyosun610@gmail.com (H.-S.K.); ysm9284@gmail.com (S.-M.Y.); kys97112@naver.com (Y.-S.G.)

2 Laboratory of Food Safety and Evaluation, Department of Biotechnology, Korea University Graduate School, Seoul 02841, Korea; cha900125@gmail.com

* Correspondence: visionkorea@korea.ac.kr (G.-J.W.); hykim@khu.ac.kr (H.-Y.K.); Tel.: +82-2-3290-3021 (G.-J.W.); +82-31-201-2123 (H.-Y.K.); Fax: +82-2-3290-3581 (G.-J.W.); +82-31-204-8116 (H.-Y.K.)

+ These authors contributed equally to the work.

Citation: Kim, E.; Shin, S.-W.; Kwak, H.-S.; Cha, M.-H.; Yang, S.-M.; Gwak, Y.-S.; Woo, G.-J.; Kim, H.-Y. Prevalence and Characteristics of Phenicol-Oxazolidinone Resistance Genes in Enterococcus Faecalis and Enterococcus Faecium Isolated from Food-Producing Animals and Meat in Korea. Int. J. Mol. Sci. 2021, 22, 11335. https://doi.org/10.3390/ ijms222111335

Academic Editor: Rustam I. Aminov

Received: 25 September 2021

Accepted: 18 October 2021

Published: 20 October 2021

Publisher's Note: MDPI stays neutral with regard to jurisdictional claims in published maps and institutional affiliations.

Copyright: (C) 2021 by the authors Licensee MDPI, Basel, Switzerland. This article is an open access article distributed under the terms and conditions of the Creative Commons Attribution (CC BY) license (https:// creativecommons.org/licenses/by/ $4.0 /)$.

\begin{abstract}
The use of phenicol antibiotics in animals has increased. In recent years, it has been reported that the transferable gene mediates phenicol-oxazolidinone resistance. This study analyzed the prevalence and characteristics of phenicol-oxazolidinone resistance genes in Enterococcus faecalis and Enterococcus faecium isolated from food-producing animals and meat in Korea in 2018. Furthermore, for the first time, we reported the genome sequence of E. faecalis strain, which possesses the phenicoloxazolidinone resistance gene on both the chromosome and plasmid. Among the 327 isolates, optrA, poxt $A$, and fexA genes were found in $15(4.6 \%), 8(2.5 \%)$, and 17 isolates (5.2\%), respectively. Twenty E. faecalis strains carrying resistance genes belonged to eight sequence types (STs), and transferability was found in 17 isolates. The genome sequences revealed that resistant genes were present in the chromosome or plasmid, or both. In strains EFS17 and EFS108, optrA was located downstream of the ermA and ant(9)-1 genes. The strains EFS36 and EFS108 harboring poxtA-encoding plasmid cocarried $f e x A$ and $c f r(D)$. These islands also contained IS1216E or the transposon Tn554, enabling the horizontal transfer of the phenicol-oxazolidinone resistance with other antimicrobial-resistant genes. Our results suggest that it is necessary to promote the prudent use of antibiotics through continuous monitoring and reevaluation.
\end{abstract}

Keywords: Enterococcus; linezolid; antibiotic resistance gene; phenicol-oxazolidinone resistance gene; optrA; fexA; poxtA

\section{Introduction}

Enterococci are symbiotic bacteria of the gastrointestinal tract of humans and animals [1]. However, enterococci infection can cause urinary tract and soft tissue infections, and in severe cases, it causes life-threatening infections such as septicemia and meningitis [2]. Enterococci of animal origin have the potential risk of transferring their antimicrobial resistance genes to human enterococci or disseminating to humans through the food chain [3]. Enterococci are also known as nosocomial pathogens, and Enterococcus species, especially Enterococcus faecalis and E. faecium, have become a particular clinical problem $[1,4,5]$. The use of florfenicol, phenicol antibiotics, has been increasing in animals to treat diseases caused by E. faecalis and E. faecium infections [5].

Linezolid, which belongs to oxazolidinone, is classified as a critically important category of antibiotics by the World Health Organization, meaning that it is a major antibiotic 
that requires prudent use [6]. Linezolid is the clinical last resort to treat drug-resistant Grampositive bacteria, including vancomycin-resistant enterococci and methicillin-resistant Staphylococcus aureus (MRSA) $[4,5,7]$. The resistance to linezolid by Gram-positive bacteria can arise through two mechanisms [8]. The first is associated with a point mutation, such as G2576T or G2505A in the 23S ribosomal ribonucleic acid (rRNA) binding site, or mutations in the genes encoding ribosomal proteins, such as L3 or L4, forming the bacterial $50 \mathrm{~S}$ subunit [7-9]. The second mechanism involves the acquisition of transferable resistance genes, such as optr $A$ and poxt $A$, encoding the adenosine triphosphate-binding cassette $\mathrm{F}$, as well as $c f r$, which encodes a methyltransferase [8]. These genes are considered as multiple resistance genes; optrA confers resistance to phenicols (chloramphenicol and florfenicol) and oxazolidinones (linezolid and tedizolid), poxt $A$ confers resistance to phenicol-oxazolidinone-tetracycline, and $c f r$ confers resistance to phenicols, oxazolidinones, lincosamides, streptogramin $\mathrm{A}$, and pleuromutilins [8]. optr $A$, poxt $A$, and $\operatorname{cfr}$ genes present as part of a plasmid or as a transposon composite; it is known that the possibility of transferring these genes to other bacteria is very high [5].

Recently, linezolid-resistant enterococci and staphylococci have been reported in patients and also in food animals. Although the distribution rate of linezolid-resistant strains is very low, recent reports on the transferable optrA gene are increasing, and multidrugresistant linezolid-resistant bacteria are emerging $[9,10]$. The optr $A$, poxt $A$, and $c f r$ encoding enterococci has been reported in Korea and many countries such as China, Italy, Ireland, and Malaysia [3]. In particular, it was confirmed that all enterococci isolated from tertiary hospital patients in Korea possessed the optrA gene, but none of them were treated with linezolid [11]. Therefore, it was estimated that the optrA-positive linezolid nonsusceptible enterococci appeared at the hospital through the community onset [11]. The optrA gene has been continuously found in enterococci from foods of animal origin in Korea, and optr $A$-encoding enterococci cocarrying the phenicol exporter gene fexA have been isolated [3]. These genes are commonly embedded in mobile genetic elements as part of plasmids or present as composite transposons in the bacterial chromosome [5]. These characteristics of mobile genetic elements enable the rapid distribution of $\operatorname{optr} A$ with $\operatorname{poxt} A$ and $c f r$ to the bacterial population. Therefore, it is important to understand the distribution of these antibiotic resistance genes.

In Korea, the use of phenicol antibiotics has steadily increased. It is reported that the amount of phenicols used as a veterinary medicinal product in 2019 is 110 tons, which is roughly twice that of 2010 [12]. This suggests that linezolid resistance is likely to increase along with the resistance to florfenicol. Florfenicol is a veterinary medicine used to control respiratory tract infections in livestock. However, since excessive use of florfenicol in animals can coselect the expression of phenicol-oxazolidinone resistance, the acquisition of cross-resistance between linezolid and florfenicol can potentially impact both humans and livestock [10]. Since this can significantly limit the treatment of multidrug-resistant bacteria, continuous monitoring and surveillance for the resistant bacteria and genes are required.

This study investigated the prevalence, antimicrobial resistance profile, and mobilizable nature of the phenicol-oxazolidinone resistance genes in E. faecalis and E. faecium isolated from food-producing animals and meat.

\section{Results}

\subsection{Identification of Phenicol-Oxazolidinone Resistant Gene}

Enterococci harboring the phenicol-oxazolidinone resistant genes optrA, poxtA, cfr, and fexA were detected from 282 E. faecalis and 45 E. faecium isolates. Among 282 E. faecalis isolates, the optrA, poxt $A$, and fex $A$ genes were identified in 15 isolates $(5.3 \%)$, six isolates $(2.1 \%)$, and 17 isolates $(6.0 \%)$, respectively, but the $c f r$ gene was not detected. In 45 E. faecium, only two strains $(4.4 \%)$ contained the poxt $A$ gene, and no other genes were identified. As shown in Table 1, the phenicol-oxazolidinone resistance genes were detected in a total of 20 E. faecalis and two E. faecium isolates originating mostly from cattle (13/22), in addition 
to pigs $(4 / 22)$ and fresh meat products including beef $(2 / 22)$ and pork (3/22). Enterococci harboring multiple phenicol-oxazolidinone resistance genes were identified. Thirteen, four, and one E. faecalis isolates were shown to carry optrA and fexA, poxtA and fexA, and optrA, $\operatorname{poxt} A$, and fexA, respectively. On the other hand, one E. faecalis had optrA only, and two of each E. faecium and $E$. faecalis isolates harbored poxt $A$ only.

Table 1. Characteristics of enterococci strains harboring phenicol-oxazolidinone resistance genes.

\begin{tabular}{|c|c|c|c|c|c|c|c|c|c|}
\hline \multirow{2}{*}{ Species } & \multirow{2}{*}{ Strain } & \multirow{2}{*}{ Source } & \multicolumn{4}{|c|}{ Detected Resistance Genes by PCR } & \multicolumn{3}{|c|}{ Transconjugants } \\
\hline & & & optrA & poxtA & $c f r$ & fexA & optrA & poxtA & fexA \\
\hline E. faecalis & EFS17 & Pork, meat & + & - & - & + & - & - & - \\
\hline E. faecalis & EFS24 & Pork, meat & + & - & - & + & + & - & + \\
\hline E. faecalis & EFS27 & Pork, meat & + & - & - & + & + & - & + \\
\hline E. faecalis & EFS36 & Beef, meat & - & + & - & + & - & + & + \\
\hline E. faecalis & EFS74 & Beef, meat & - & + & - & - & - & + & - \\
\hline E. faecalis & EFS98 & Pig, slaughterhouse & - & + & - & + & - & - & - \\
\hline E. faecalis & EFS99 & Pig, slaughterhouse & - & + & - & + & - & - & + \\
\hline E. faecalis & EFS108 & Pig, slaughterhouse & + & + & - & + & - & + & + \\
\hline E. faecalis & EFS117 & Cattle, slaughterhouse & + & - & - & - & - & - & - \\
\hline E. faecalis & EFS147 & Cattle, farm & + & - & - & + & + & - & + \\
\hline E. faecalis & EFS151 & Cattle, farm & + & - & - & + & + & - & + \\
\hline E. faecalis & EFS153 & Cattle, farm & + & - & - & + & + & - & + \\
\hline E. faecalis & EFS154 & Cattle, farm & + & - & - & + & + & - & + \\
\hline E. faecalis & EFS158 & Cattle, farm & + & - & - & + & + & - & + \\
\hline E. faecalis & EFS253 & Pig, slaughterhouse & + & - & - & + & + & - & + \\
\hline E. faecalis & EFS255 & Cattle, slaughterhouse & - & + & - & - & - & + & - \\
\hline E. faecalis & EFS268 & Cattle, farm & + & - & - & + & + & - & + \\
\hline E. faecalis & EFS269 & Cattle, farm & + & - & - & + & + & - & + \\
\hline E. faecalis & EFS270 & Cattle, farm & + & - & - & + & + & - & + \\
\hline E. faecalis & EFS271 & Cattle, farm & + & - & - & + & + & - & + \\
\hline E. faecium & EFM21 & Cattle, farm & - & + & - & - & - & - & - \\
\hline E. faecium & EFM262 & Cattle, farm & - & + & - & - & - & - & - \\
\hline
\end{tabular}

+ , indicates the presence of phenicol-oxazolidinone resistance genes or transferability; -, indicates absence of resistance genes or not transferability.

\subsection{Antimicrobial-Resistant Pattern}

Twenty-two enterococcal strains with phenicol-oxazolidinone resistance genes showed the highest resistance to quinupristin/dalfopristin $(100 \%)$, florfenicol $(100 \%)$, tetracycline $(95 \%)$, erythromycin $(95 \%)$, tylosin $(95 \%)$, streptomycin $(77 \%)$, chloramphenicol $(77 \%)$, and kanamycin $(64 \%)$, while the antimicrobial resistance level was low against gentamicin $(14 \%)$, ciprofloxacin $(9 \%)$, daptomycin $(9 \%)$, tigecycline $(5 \%)$, and ampicillin $(5 \%)$. Linezolid-resistant enterococci were identified in three isolates (14\%), and no vancomycin or salinomycin resistance was detected. Except for one strain, 21 strains (19 E. faecalis and two E. faecium) showed a multidrug resistance pattern (Table 2).

The minimum inhibitory concentration (MIC) of three linezolid-resistant strains was confirmed to be $8 \mathrm{mg} / \mathrm{L}$, and the MIC of five linezolid-intermediate strains was determined to be $4 \mathrm{mg} / \mathrm{L}$ (Table 2). Among the florfenicol-resistant strains, a breakpoint of $16 \mathrm{mg} / \mathrm{L}$ or higher was confirmed in 21 strains, and of these, seven strains had a high MIC of $64 \mathrm{mg} / \mathrm{L}$.

\subsection{Molecular Typing by Multilocus Sequence Typing (MLST)}

As shown in Table 3, the MLST analysis of 20 E. faecalis isolates revealed eight sequence types (STs) including ST593, ST100, ST16, ST585, ST915, ST338, ST47, and ST27. ST593 (clonal complex (CC) singleton) was the most predominant with nine strains (43\%), and ST100 (CC100) and ST16 (CC16) were determined from three strains each, whilst ST585 (CC4), ST 915 (CC915), ST338 (CC4), ST47 (CC47), and ST27 (CC27) were each identified from a strain. The goeBURST analysis grouped all available STs into six CC and identified 
ST593, the most prevalent ST type in this study, as a singleton ST, as shown in the minimum spanning tree in Figure 1.

Table 2. Antimicrobial resistance patterns of enterococci strains harboring phenicol-oxazolidinone resistance genes.

\begin{tabular}{|c|c|c|c|c|}
\hline \multirow{2}{*}{ Scheme 1.} & \multirow{2}{*}{ Strain } & \multirow{2}{*}{ Antimicrobial Resistance Pattern ${ }^{1}$} & \multicolumn{2}{|c|}{$\operatorname{MIC}^{2}(\mathrm{mg} / \mathrm{L})$} \\
\hline & & & LZD & FFN \\
\hline E. faecalis & EFS17 & TET, CIP, ERY, TYLT, LZD, GEN, KAN, STR, CHL, FFN & 8 & 64 \\
\hline E. faecalis & EFS24 & TET, ERY, TYLT, LZD, GEN, KAN, STR, CHL, FFN & 8 & 64 \\
\hline E. faecalis & EFS27 & TET, ERY, TYLT, KAN, STR, CHL, FFN & 4 & 64 \\
\hline E. faecalis & EFS36 & ERY, TYLT, GEN, KAN, STR, CHL, FFN & 2 & 64 \\
\hline E. faecalis & EFS74 & TET, FFN & 4 & 16 \\
\hline E. faecalis & EFS98 & TET, ERY, TYLT, STR, FFN & 4 & 64 \\
\hline E. faecalis & EFS99 & TET, ERY, TYLT, STR, FFN & 4 & 16 \\
\hline E. faecalis & EFS108 & TET, ERY, TYLT, CHL, FFN & 2 & 64 \\
\hline E. faecalis & EFS117 & TET, DAP, ERY, TYLT, FFN & - & 16 \\
\hline E. faecalis & EFS147 & TET, ERY, TYLT, CHL, FFN & - & 32 \\
\hline E. faecalis & EFS151 & TET, ERY, TYLT, KAN, STR, CHL, FFN & - & 32 \\
\hline E. faecalis & EFS153 & TET, ERY, TYLT, KAN, STR, CHL, FFN & - & 32 \\
\hline E. faecalis & EFS154 & TET, ERY, TYLT, STR, CHL, FFN & - & 32 \\
\hline E. faecalis & EFS158 & TET, ERY, TYLT, KAN, STR, CHL, FFN & - & 32 \\
\hline E. faecalis & EFS253 & TET, ERY, TYLT, LZD, GEN, KAN, STR, CHL, FFN & 8 & 64 \\
\hline E. faecalis & EFS255 & TET, ERY, TYLT, FFN & - & 16 \\
\hline E. faecalis & EFS268 & TET, ERY, TYLT, KAN, STR, CHL, FFN & - & 32 \\
\hline E. faecalis & EFS269 & TET, ERY, TYLT, KAN, STR, CHL, FFN & - & 32 \\
\hline E. faecalis & EFS270 & TET, ERY, TYLT, KAN, STR, CHL, FFN & - & 32 \\
\hline E. faecalis & EFS271 & TET, TGC, ERY, TYLT, KAN, STR, CHL, FFN & - & 32 \\
\hline E. faecium & EFM21 & TET, CIP, DAP, ERY, TYLT, KAN, STR, CHL, FFN & 4 & 32 \\
\hline E. faecium & EFM262 & TET, TGC, CIP, ERY, TYLT, KAN, STR, AMP, CHL, FFN & - & 32 \\
\hline
\end{tabular}

${ }^{1}$ AMP, ampicillin; CHL, chloramphenicol; CIP, ciprofloxa-cin; DAP, daptomycin; ERY, erythromycin; FFN, florfenicol; GEN, gentamycin; KAN, kanamycin; LZD, linezolid; STR, streptomycin; TET, tetracycline; TGC, tigecycline; TYLT, tylosin; ${ }^{2}$ MIC, minimum inhibitory concentration; LNZ, linezolid; FFN, florfenicol.

Table 3. MLST analysis of enterococci strains harboring phenicol-oxazolidinone resistance genes.

\begin{tabular}{|c|c|c|c|c|c|c|c|c|c|}
\hline \multirow{2}{*}{ Strain } & \multicolumn{7}{|c|}{ Allele } & \multirow{2}{*}{$\mathrm{ST}^{1}$} & \multirow{2}{*}{$\mathrm{CC}^{2}$} \\
\hline & $g d h$ & $g y d$ & pstS & $g k i$ & aroE & $x p t$ & yiqL & & \\
\hline EFS17 & 8 & 7 & 7 & 4 & 4 & 4 & 1 & 585 & 4 \\
\hline EFS24 & 5 & 1 & 1 & 3 & 7 & 7 & 6 & 16 & 16 \\
\hline EFS27 & 39 & 2 & 49 & 45 & 7 & 2 & 17 & 915 & 915 \\
\hline EFS36 & 8 & 7 & 7 & 5 & 4 & 14 & 1 & 338 & 4 \\
\hline EFS74 & 19 & 1 & 24 & 22 & 19 & 17 & 14 & 47 & 47 \\
\hline EFS98 & 34 & 2 & 17 & 37 & 29 & 23 & 6 & 100 & 100 \\
\hline EFS99 & 34 & 2 & 17 & 37 & 29 & 23 & 6 & 100 & 100 \\
\hline EFS108 & 3 & 2 & 7 & 10 & 10 & 2 & 7 & 27 & 27 \\
\hline EFS117 & 5 & 1 & 1 & 3 & 7 & 7 & 6 & 16 & 16 \\
\hline EFS147 & 14 & 2 & 17 & 1 & 3 & 3 & 17 & 593 & Singleton \\
\hline EFS151 & 14 & 2 & 17 & 1 & 3 & 3 & 17 & 593 & Singleton \\
\hline EFS153 & 14 & 2 & 17 & 1 & 3 & 3 & 17 & 593 & Singleton \\
\hline EFS154 & 14 & 2 & 17 & 1 & 3 & 3 & 17 & 593 & Singleton \\
\hline EFS158 & 14 & 2 & 17 & 1 & 3 & 3 & 17 & 593 & Singleton \\
\hline EFS253 & 5 & 1 & 1 & 3 & 7 & 7 & 6 & 16 & 16 \\
\hline EFS255 & 34 & 2 & 17 & 37 & 29 & 23 & 6 & 100 & 100 \\
\hline EFS268 & 14 & 2 & 17 & 1 & 3 & 3 & 17 & 593 & Singleton \\
\hline EFS269 & 14 & 2 & 17 & 1 & 3 & 3 & 17 & 593 & Singleton \\
\hline EFS270 & 14 & 2 & 17 & 1 & 3 & 3 & 17 & 593 & Singleton \\
\hline EFS271 & 14 & 2 & 17 & 1 & 3 & 3 & 17 & 593 & Singleton \\
\hline
\end{tabular}

${ }^{1} \mathrm{ST}$, sequence type; ${ }^{2} \mathrm{CC}$, clonal complex. 


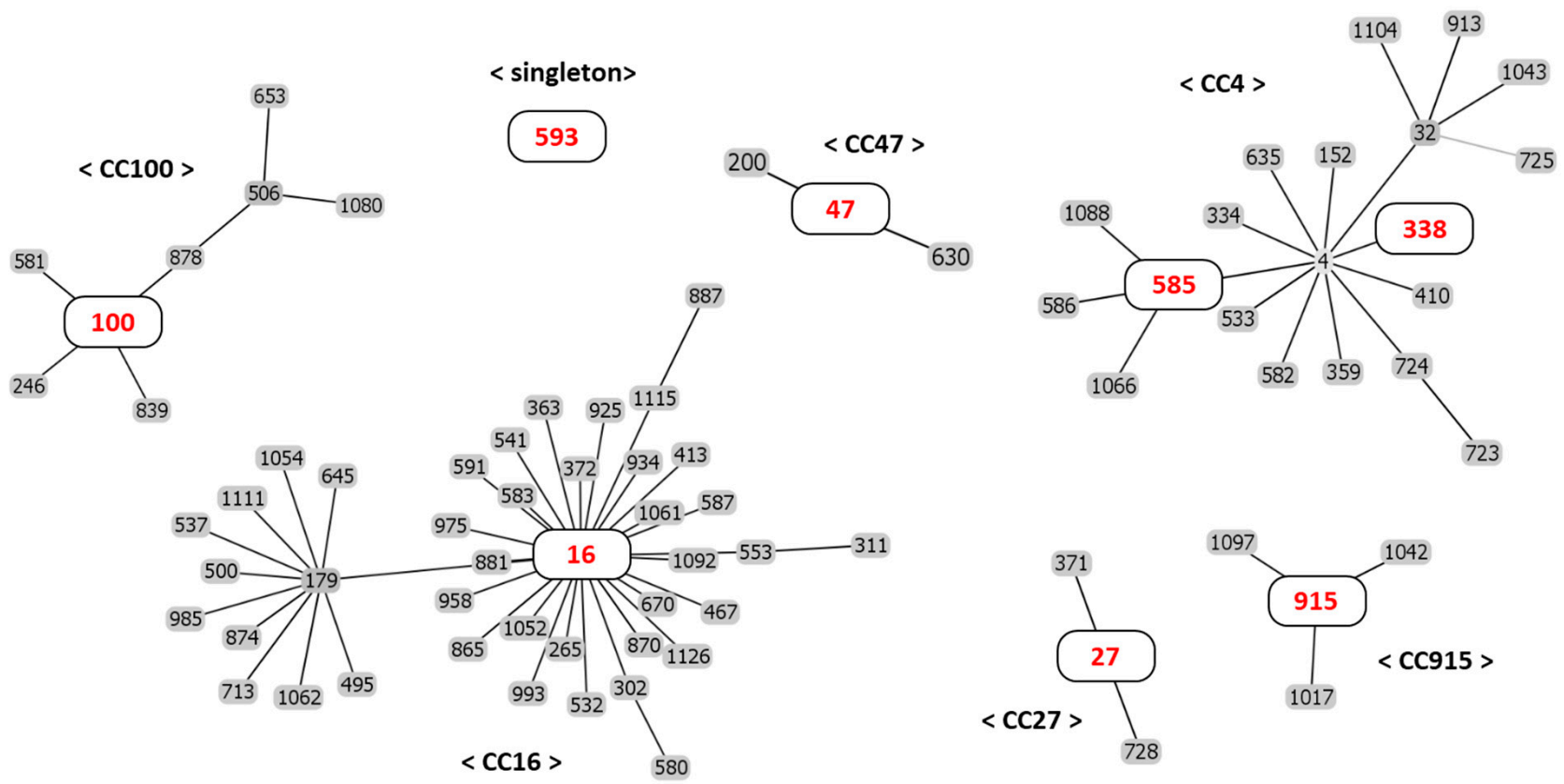

Figure 1. Graphical presentation of multilocus sequence typing data. Each ST analyzed in this study marked in red. The lines connect single-locus variants. The clonal relatedness between sequence types (STs) was analyzed against the entire E. faecalis database. Clonal complexes (CCs) are indicated. Eight sequence types (STs) were identified among the 20 E. faecalis isolates: ST593, ST100, ST16, ST27, ST338, ST47, ST585, and ST915. The eBURST algorithm clustered the STs into one singleton and six clonal complexes: CC100, CC16, CC27, CC4, CC47, and CC915.

\subsection{Transferability of Phenicol-Oxazolidinone Resistance Genes}

The transferability of optrA, poxtA, and fexA genes was tested by the broth-mating method. It was confirmed that the resistance gene was transferred in 17 out of $22(77 \%)$ enterococcal isolates. In two E. faecium strains, gene transfer was not identified. As shown in Table 1, each of the optrA, poxtA, and fexA genes possessed by each donor strain was successfully delivered to the recipient strain.

\subsection{Complete Genome Sequencing}

\subsubsection{Genome Features of Three Isolates}

Complete genome sequencing was performed to identify the underlying antimicrobial resistance mechanisms. Genome analysis showed that the EFS17 strain consists of one chromosome and one plasmid, and EFS36 and EFS108 strains had two and five plasmids, respectively (Table 4). The genomic sizes of the strains EFS17, EFS36, and EFS108 consisted of 2,914,315 bp, 3,095,754 bp, and 3,093,936 bp with a GC content of $37.4 \%, 37.3 \%$, and $37.3 \%$, respectively. The genomic features of the EFS17 strain included 2803 coding genes and 73 coding regions for RNAs, of which 61 were transfer RNAs (tRNAs). Meanwhile, the EFS36 genome contained 3057 coding sequences and 78 coding regions for RNAs, including 66 tRNAs. The EFS108 genome displayed 2980 coding genes and 73 coding regions for RNAs, of which 61 were tRNAs.

\subsubsection{In Silico Identification of Antimicrobial Resistance Genes}

A blastn search for known resistance genes revealed that three strains possessed the phenicol-oxazolidinone resistance optrA gene or poxt $A$ gene; two strains also carried the $c f r(D)$ gene (Figure 2). EFS17 and EFS108 strains harbored the optrA gene, whereas EFS36 and EFS108 strains had the $c f r(D)$ and poxt $A$ genes. The phenicol-oxazolidinone resistance genes were present on the chromosome in the EFS17 strain; for the EFS36 strain, this gene was present on the plasmid (pEFS36_2). In the EFS108 strain, phenicol-oxazolidinone resistance genes were present in both the chromosome and plasmid (pEFS108_1) (Figure 2). 
Table 4. Genomic features of three E. faecalis isolates.

\begin{tabular}{cccc}
\hline Genomic Features & EFS17 & EFS36 & EFS108 \\
\hline Genome size (bp) & $2,914,315$ & $3,095,754$ & $3,093,936$ \\
Chromosome size (bp) & $2,838,954$ & $3,016,592$ & $2,816,588$ \\
Number of plasmids & 1 & 2 & 5 \\
Size range of plasmid(s) (bp) & 75,361 & $35,757-43,405$ & $5120-97,455$ \\
GC content (\%) & 37.4 & 37.3 & 37.3 \\
Number of genes & 2876 & 3135 & 3053 \\
Number of coding genes & 2803 & 3057 & 2980 \\
Coding genes in chromosome & 2712 & 2964 & 2670 \\
Coding genes in plasmid(s) & 91 & 93 & 310 \\
Number of RNAs & 73 & 78 & 73 \\
\hline
\end{tabular}

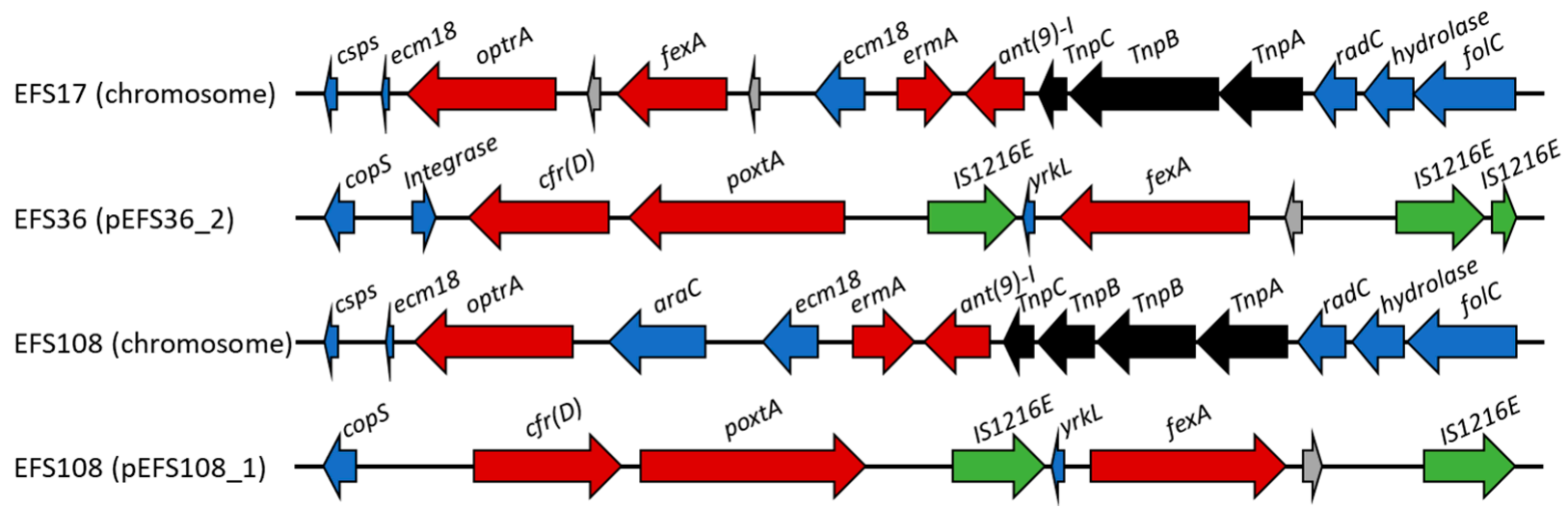

Figure 2. Schematic representation of phenicol-oxazolidinone resistance genes loci in three E. faecalis genomes. Genes and their orientation are shown with arrows and labeled; red, green, black, blue, and gray indicate antibiotic resistance gene, insertion sequences (ISs), transposase, known proteins, and hypothetical proteins, respectively. Csps, cold shock protein of CSP family; ecm18, class I SAM-dependent methyltransferase; radC, DNA repair protein RadC; folC, dihydrofolate synthase; yrkL, NAD(P)H oxidoreductase YRKL.

The EFS17 strain harbored optrA and fexA phenicol resistance genes. In the EFS17 strain, the fexA gene was encoded 689 bp upstream of the optrA gene, whereas the EFS108 strain lacked fexA (Table S1). Transposon Tn554 was detected downstream of optrA in EFS17 and EFS108 strains (Figure 2). Moreover, the ermA and ant(9)-I genes conferring resistance to erythromycin and spectinomycin antibiotic were located upstream of the optrA genes in both EFS17 and EFS108 isolates. Additionally, analysis of complete genome sequence revealed that the EFS17 strain included additional genes for resistance to macrolide-lincosamide-streptogramin B (ermA, ermB, lunB, las A, and lasE), aminoglycosides $\left(\operatorname{aac}\left(6^{\prime}\right)-\operatorname{aph}\left(2^{\prime \prime}\right), \operatorname{aph}\left(3^{\prime}\right)\right.$-III, str, and ant (6)-Ia), and tetracyclines (tet $M$ and tetL) (Table 5). EFS36 and EFS108 strains harbored a poxtA-carrying plasmid named pEFS36_2 and pEFS108_1, respectively (Figure 3). Plasmid pEFS36_2 was 35,757 bp in length with a GC content of $34.3 \%$. Plasmid pEFS108_1 was 97,455 bp in length with a GC content of $33.8 \%$. These plasmids coharbor $f e x A, c f r(D)$, and IS1216E, which were identified in two or three places. Therefore, EFS17, EFS36, and EFS108 contained several mobile gene elements and transposase-associated genes, enabling the horizontal transfer of the phenicoloxazolidinone resistance genes. 
Table 5. Antimicrobial resistance genes in three E. faecalis strains.

\begin{tabular}{|c|c|c|}
\hline Strain & Class & Antimicrobial Resistance Genes \\
\hline \multirow[t]{12}{*}{ EFS17 } & aminocyclitol & $\operatorname{ant}(9)-I a$ \\
\hline & aminoglycoside & $\operatorname{aac}\left(6^{\prime}\right)-\operatorname{aph}\left(2^{\prime \prime}\right), \operatorname{aph}\left(3^{\prime}\right)-I I I$, str, ant (6)-Ia \\
\hline & fluoroquinolone & $\operatorname{parC}$ \\
\hline & folate pathway antagonist & $d f r G$ \\
\hline & lincosamide & lsa $A, l_{s} a E, \ln u B$, erm $A$, erm $B$ \\
\hline & macrolide & $\operatorname{erm} A, \operatorname{erm} B$ \\
\hline & oxazolidinone & optrA \\
\hline & phenicol & fexA, optrA, cat \\
\hline & pleuromutilin & lsaE \\
\hline & streptogramin a & $l s a A, l s a E$ \\
\hline & streptogramin $b$ & ermA, ermB \\
\hline & tetracycline & tet $M$, tet $L$ \\
\hline \multirow[t]{9}{*}{ EFS36 } & aminoglycoside & $\operatorname{aac}\left(6^{\prime}\right)-\operatorname{aph}\left(2^{\prime \prime}\right), \operatorname{aph}\left(3^{\prime}\right)-I I I, \operatorname{ant}(6)-I a$ \\
\hline & lincosamide & $\operatorname{ls} a E, \operatorname{cfr}(D), \ln u B, \operatorname{ls} a A$, ermB \\
\hline & macrolide & ermB \\
\hline & oxazolidinone & $c f r(D), \operatorname{poxt} A$ \\
\hline & phenicol & $f e x A, \operatorname{poxt} A, c f r(D)$ \\
\hline & pleuromutilin & $l s a E, c f r(D)$ \\
\hline & streptogramin a & $l s a A, l s a E, c f r(D)$ \\
\hline & streptogramin b & ermB \\
\hline & tetracycline & poxtA \\
\hline \multirow[t]{8}{*}{ EFS108 } & aminocyclitol & ant(9)-Ia \\
\hline & lincosamide & $\operatorname{erm} A$, erm $B$, lsaA \\
\hline & macrolide & erm $A$, erm $B$ \\
\hline & oxazolidinone & optrA, $\operatorname{cfr}(D), \operatorname{poxt} A$ \\
\hline & phenicol & fexA, optr $A$, cat \\
\hline & streptogramin a & lsaA \\
\hline & streptogramin $b$ & erm $A$, erm $B$ \\
\hline & tetracycline & tetM, tetL \\
\hline
\end{tabular}
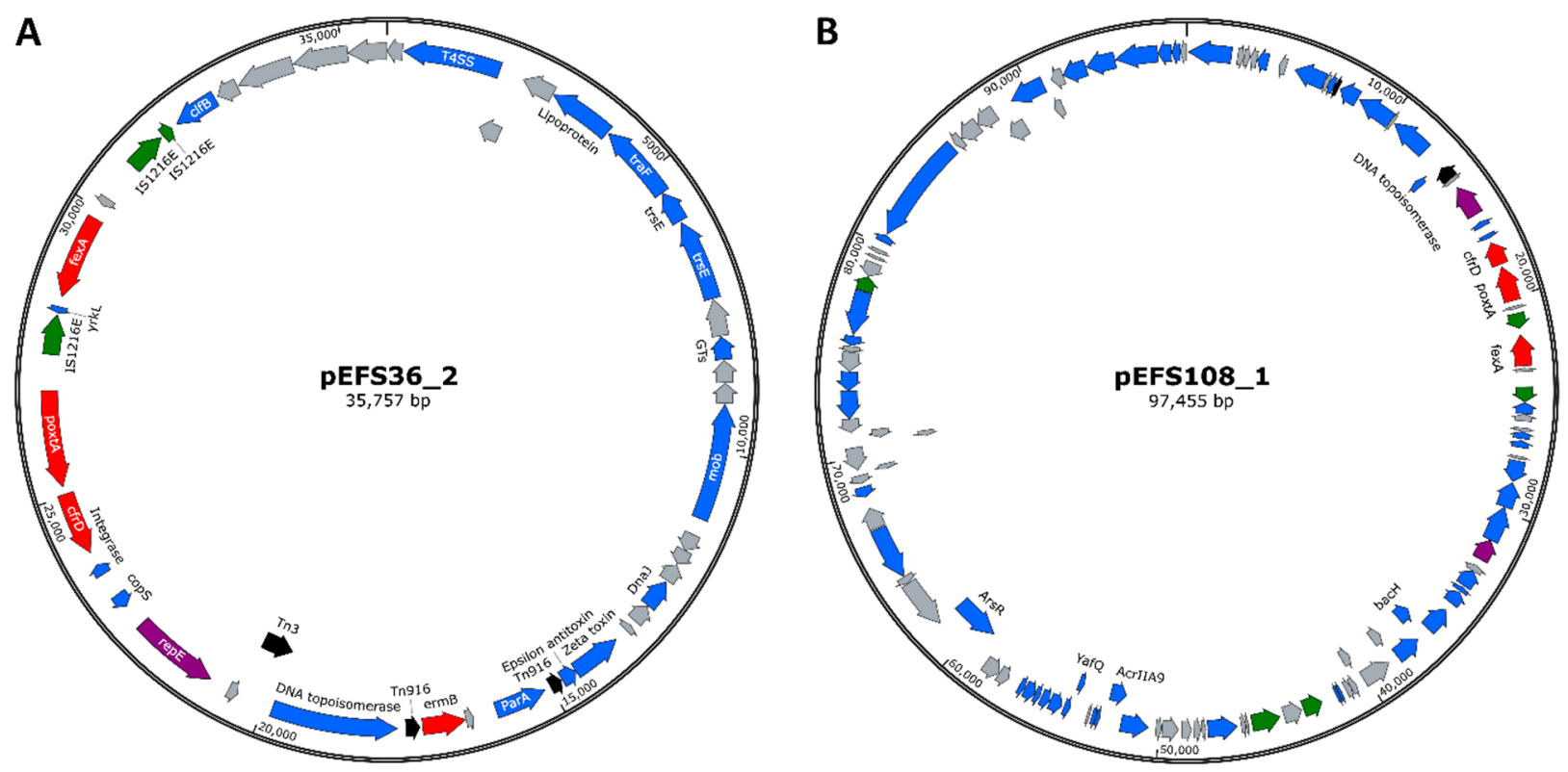

Figure 3. Schematic diagram of genetic structure of poxt $A$ linezolid resistance gene-containing plasmids (A) pEFS36_2 from EFS36 isolate and (B) pEFS108_1 from EFS108 isolate. Genes and their orientation are indicated by arrow as follows: red, green, black, purple, blue, and gray represent antibiotic resistance gene, ISs, transposase, rep protein, other protein, and hypothetical proteins, respectively. 


\section{Discussion}

Knowledge of the distribution of antimicrobial-resistant strains in the food chain and food animals is important in determining the potential risk to human health [13]. Here, we investigated the prevalence and genetic characterization of phenicol-oxazolidinone resistance genes to understand better the phenicol-oxazolidinone resistance profiles of enterococci isolates obtained from food animals, animal carcass, and meat in Korea. Moreover, for the first time in Korea, this study reports the complete genome sequence of the E. faecalis EFS108 strain harboring phenicol-oxazolidinone resistant genes on both the chromosome and the plasmid.

Overall, 6.7\% (22/327) of the enterococci isolates tested harbored phenicol-oxazolidinone resistance genes. Among them, optrA, poxtA, and fexA were detected in 15 isolates (4.6\%), 8 isolates $(2.5 \%)$, and 17 isolates $(5.2 \%)$, respectively, confirming that these genes were distributed at a low level. Consistent with this study, a low phenicol-oxazolidinone resistance rate was reported in E. faecalis and E. faecium from food animals and animal carcasses in Korea [1,3,14,15], China [16], and Europe [17]. In a previous study, enterococci strains with phenicol-oxazolidinone resistance genes were isolated at slightly higher rates in E. faecalis than E. faecium, and the optrA gene was confirmed only in E. faecalis isolates [18]. Our results are congruent with reports from previous studies where optrA is predominantly present in E. faecalis, and the reason is presumed that the loss of conjugated resistance plasmids occurs in E. faecium [4,19]. Moreover, none of the phenicol-oxazolidinone resistant enterococci strains carried the $c f r$ gene, but the $c f r(D)$ gene was confirmed through complete genome sequencing in two E. faecalis isolates (EFS36 and EFS108). Therefore, when monitoring the phenicol-oxazolidinone resistance gene, it will be necessary to examine the cfr gene and its variants. The reason is that horizontal gene transfer is possible between $c f r$ gene-carrying strains. In addition to the $c f r$ gene, variants $c f r(B), c f r(C)$, and $c f r(D)$ have been reported in clinical isolates $[9,10]$.

Twenty-two enterococcal isolates harboring phenicol-oxazolidinone resistance genes were resistant to one or more antimicrobial agents. Multidrug-resistant isolates were observed in both E. faecalis (95\%) and E. faecium (100\%) with high frequencies. A high resistance rate was observed for quinupristin/dalfopristin, florfenicol, tetracycline, erythromycin, and tylosin. Antimicrobial patterns were similar to those of other food animals. The high-level resistance to phenicols, tetracycline, and macrolides (erythromycin and tylosin) was also found in food animals and their carcass in Korea [1] and European countries [20]. These antimicrobial agents are generally administered to food animals in Korea [1]. Resistance to linezolid was observed in three E. faecalis isolates but not in E. faecium. All linezolid-resistant $E$. faecalis isolates were also resistant to florfenicol and chloramphenicol.

In the MLST analysis to investigate the clonal relationship of phenicol-oxazolidinone resistance strains, the STs of enterococci isolated from meat, slaughterhouses, and farms could be classified into eight different types. Consistent with this study, the diversity of STs was also reported in enterococci isolated from food animals and animal carcass in Korea [3,8] and humans and animals in China [21]. Some E. faecalis strains isolated from different samples had optrA and fexA genes commonly, and the ST was the same as 593. From this result, resistance could be acquired through antibiotics for animals such as florfenicol in the farm. It can be estimated that resistance genes optrA and fexA may be spread to animals within the farm through feed or the farm environment [1]. ST16 was identified in three optrA-positive E. faecalis; two isolates cocarried fexA, and one isolate has only optrA. This ST has been identified in food animals and humans in Korea and other countries and is known as an optrA-carrying clone with a worldwide distribution [11,19]. In addition, EFS17 isolated from pork was identified as ST585, which coharbored optrA and fexA. ST585 E. faecalis harboring optrA has also been reported in the United States, China, and Germany [11].

Complete genome sequencing was performed on three E. faecalis isolates (EFS17, EFS36, and EFS108), which have different combinations of phenicol resistance genes, 
to investigate the location and genetic environment of optrA and poxtA. According to a report investigating the genetic environment of optrA-carrying plasmid in E. faecalis originated from humans and animals, the IS1216E element was detected either upstream or downstream of the optrA gene [22]. In addition, fexA and $\operatorname{erm}(A)$-related genes were found upstream and downstream of optrA, respectively [22]. Conversely, when optrA is located in the chromosome, the optrA gene was located immediately downstream of the transcriptional regulator gene $\mathrm{araC}$ or downstream of the fex $A$ gene [22]. Alternatively, the integration of the optrA gene was observed between Tn558 and Tn554 relics located in the chromosomal radC gene [22]. The $\mathrm{radC}$ gene is the common integration site for transposons of the Tn554 family [22]. Our study found that the optrA gene was located on the EFS17 and EFS108 genomes but not in the EFS36 genome. As in a previous study [15], the optrA gene on the EFS108 chromosome was identified downstream of araC, whereas the optrA gene on the EFS17 chromosome was found downstream of fexA. Moreover, the chromosomal $\mathrm{radC}$ gene was found in both EFS17 and EFS108.

Phenicol-oxazolidinone resistance has been reported to be mediated by the transferable phenicol-oxazolidinone resistance gene in recent years $[1,16]$. The optrA gene in strains EFS36 and EFS108 was located adjacent to Tn554 transposon. Tn554 was also found upstream of the optrA gene in other Enterococcus strains and Staphylococcus sciuri [23]. The functionally active Tn554 can be excised from their host DNA and generate circular forms that precede the integration of the transposon into a target sequence $[23,24]$. A similar genetic arrangement of optrA and Tn554 was identified in enterococci and staphylococci $[13,16]$, suggesting that optr $A$ can be mediated for transfer between different bacterial genus and species. The optrA gene was flanked by transposon or insertion sequences, indicating that a mobile genetic element mediates the horizontal transfer of optrA between bacteria of different genera, which should be given more attention to avoid disseminating the oxazolidinone resistance gene in the environment.

The poxt $A$ gene was located on plasmids in two enterococci strains. The poxt $A$ gene is often surrounded by bacteria insertion sequences on the plasmid in staphylococci or enterococci strains $[13,16]$. Our data showed that all poxt $A$ found on plasmids were flanked by IS1216E, similar to the previous studies $[9,16,25]$. IS1216E belongs to the IS6 family of the bacteria insertion sequence, which among other things mediates transmission of the macrolide-lincosamide-streptogramin B resistance genes erm $(B)$ in E. hirae, the oxazolidinone resistance gene cfr in E. faecalis, vancomycin resistance gene vanA in E. faecium, and tetracycline resistance gene tet $S$ in Streptococcus infantis [23,26-28]. This indicates that poxt $A$ can be transferred between different bacterial genera by IS-mediated recombination events, which plays an important role in disseminating antimicrobial resistance genes. Furthermore, two plasmids (pEFS36_2 and pEFS108_1) carrying poxt $A$ gene also coharbored other resistance genes, such as fexA and $c f r(D)$. This result suggests that poxt $A$ together with fex $A$ and $c f r(D)$ genes may be disseminated among different strains via IS1216E.

The transferability of phenicol-oxazolidinone resistance genes was confirmed through an experiment by broth mating and a genetic context analysis using complete genome sequencing. The transferable phenicol-oxazolidinone resistant genes, optrA and poxt $A$, were detected by the broth mating method in 12 and four E. faecalis isolates. All of them, except for two isolates harboring poxtA, cocarried the phenicol exporter gene fexA. These results were also confirmed by whole-genome sequencing analysis. Genome analysis showed that transposons or insertion sequences mediating resistance genes were adjacent to optrA, poxt $A$, and fexA genes.

In conclusion, although the occurrence of a phenicol-oxazolidinone resistance gene in enterococci is still rare among food animals, a high rate of transferable phenicoloxazolidinone genes was observed in these strains. The emergence of these genes in enterococci isolates from food animals is a serious problem, as they can be transmitted to humans through the food chain. The spread of these genes can significantly limit the treatment against multidrug-resistant bacteria. Hence, active surveillance of phenicol- 
oxazolidinone-resistant bacteria and related resistance genes is essential to prevent the spread of resistant enterococci isolates.

\section{Materials and Methods}

\subsection{Enterococcus Strains}

In 2018, 282 E. faecalis strains and 45 E. faecium strains were isolated in Korea from 43 meat (19 beef and 24 pork), 24 slaughterhouses, and 16 farms. In detail, 128 E. faecalis and 9 E. faecium strains were isolated from meat, and $48 \mathrm{E}$. faecalis and $13 \mathrm{E}$. faecium strains were isolated from the slaughterhouse. The remaining 106 E. faecalis and 23 E. faecium strains were isolated from the farm. All isolates were used in the experiment after being identified as enterococci using VITEK ${ }^{\circledR}$ MS (bioMérieux, Marcy l'Etoile, France). All enterococci strains were cultured on tryptone soya agar (TSA) medium at $37^{\circ} \mathrm{C}$ for $24 \mathrm{~h}$.

\subsection{Screening of Phenicol-Oxazolidinone Resistance Gene by Polymerase Chain Reaction (PCR)}

Genomic DNA from Enterococcus isolates was extracted using the DNeasy Blood \& Tissue kit (Qiagen, Hilden, Germany) according to the manufacturer's instructions. The presence or absence of the phenicol-oxazolidinone resistance genes, such as optrA, poxt $A, c f r$, and fex $A$, in the isolates was detected by PCR according to the previous studies $[9,24]$. The primer sequences of each gene are shown in Table 6. The PCR mixture $(25 \mu \mathrm{L})$ contained $1 \mu \mathrm{L}$ of each primer $(0.4 \mu \mathrm{M}), 0.1 \mu \mathrm{L}$ of Taq polymerase $(5 \mathrm{U} / \mu \mathrm{L}), 2.5 \mu \mathrm{L}$ of $10 \times$ buffer, $16.4 \mu \mathrm{L}$ of distilled water, and $2 \mu \mathrm{L}$ of template DNA. Amplification for the optrA, poxtA, and $c f r$ genes was performed at $95^{\circ} \mathrm{C}$ for $2 \mathrm{~min}$, and 25 cycles of $95{ }^{\circ} \mathrm{C}$ for $15 \mathrm{~s}, 53{ }^{\circ} \mathrm{C}$ for $15 \mathrm{~s}, 68^{\circ} \mathrm{C}$ for $1 \mathrm{~min}$, and final elongation at $68^{\circ} \mathrm{C}$ for $5 \mathrm{~min}$. PCR conditions for the fexA gene were as follows: initial denaturation at $94^{\circ} \mathrm{C}$ for $5 \mathrm{~min}, 35$ cycles at $94{ }^{\circ} \mathrm{C}$ for $1 \mathrm{~min}$, $57^{\circ} \mathrm{C}$ for $1 \mathrm{~min}, 72{ }^{\circ} \mathrm{C}$ for $1.5 \mathrm{~min}$, and final elongation at $72{ }^{\circ} \mathrm{C}$ for $5 \mathrm{~min}$. The PCR-positive control DNA for optrA, poxtA, cfr, and fexA was kindly provided from the Animal and Plant Quarantine Agency in Korea.

Table 6. Primer sequences for the detection of phenicol-oxazolidinone resistance genes.

\begin{tabular}{|c|c|c|c|c|}
\hline Target & Primer & Nucleotide Sequence $\left(5^{\prime} \rightarrow 3^{\prime}\right)$ & Amplicon (bp) & Reference \\
\hline \multirow[t]{2}{*}{$c f r$} & cfr-F & TGC TAC AGG CGA CAT TGG AT & 137 & [9] \\
\hline & cfr-R & GAC GGT TGG CTA GAG CTT CA & & \\
\hline \multirow[t]{2}{*}{ optrA } & optrA-F & ACC GGT GTC CTC TTT GTC AG & 369 & [9] \\
\hline & optrA-R & TCA ATG GAG TTA CGA TCG CCT T & & \\
\hline \multirow[t]{2}{*}{$\operatorname{poxt} A$} & poxtA-F & TCA GAG CCG TAC TGA GCA AC & 167 & [9] \\
\hline & poxtA-R & CGT TTC TGG GTC AAG GTG GT & & \\
\hline \multirow[t]{2}{*}{ fexA } & fexA-F & GTA CTT GTA GGT GCA ATT ACG GCT GA & 1272 & [24] \\
\hline & fexA-R & CGC ATC TGA GTA GGA CAT AGC GTC & & \\
\hline
\end{tabular}

\subsection{Antimicrobial Susceptibility Testing}

An antimicrobial susceptibility test was performed on the 22 E. faecalis and E. faecium isolates harboring at least one of the phenicol-oxazolidinone resistance genes optr $A$, poxtA, $c f r$, and fexA. The MIC of 16 antimicrobial agents was determined by the broth microdilution method using commercially available Sensititre1 panel KRVP2F (TREK Diagnostic Systems, West Sussex, UK), according to the manufacturer's instructions. Staphylococcus aureus ATCC 29213 and E. faecalis ATCC 29212 were used as the quality control strains. The interpretation of the results followed the Clinical and Laboratory Standards Institute guidelines [29]. Sixteen antibiotics used in this study were as follows: gentamycin (GEN), streptomycin (STR), kanamycin (KAM), ampicillin (AMP), ciprofloxacin (CIP), vancomycin (VAN), tigecycline (TGC), erythromycin (ERY), tylosin (TYLT), linezolid (LZD), chloramphenicol (CHL), florfenicol (FFN), quinupristin/dalfopristin (SYN), tetracycline (TET), daptomycin (DAP), and salinomycin (SAL). 


\subsection{MLST Analysis}

MLST analysis of $20 \mathrm{E}$. faecalis isolates harboring phenicol-oxazolidinone resistance genes was performed according to a previous study [30]. Seven housekeeping genes, such as $g d h, g y d, p s t S, g k i$, aroE, xpt, and yqiL, were amplified by PCR and then sequenced. Sequence types of E. faecalis were determined using the E. faecalis MLST database in PubMLST (http:/ / pubmlst.org/efaecalis/, accessed on 22 September 2021). The clonal relationship between STs was analyzed by the goeBURST algorithm using the PHYLOViZ software v 2.0 [31].

\subsection{Conjugation}

Twenty-two E. faecalis and E. faecium isolates harboring phenicol-oxazolidinone resistance genes were used as the donor cells, and with Escherichia coli strain J53 served as the recipient strain for the conjugation experiments using methods previously described by Aljahdali et al. with minor modifications [32]. Briefly, each donor and recipient cells were cultured on TSA agar and incubated at $37^{\circ} \mathrm{C}$ for $24 \mathrm{~h}$. Cultured donor and recipient cells were mixed in $500 \mu \mathrm{L} \mathrm{LB}$ broth $(1: 1)$ and incubated at $37^{\circ} \mathrm{C}$ for $3 \mathrm{~h}$. Transconjugants were streaked on LB agar containing $200 \mathrm{mg} / \mathrm{L}$ sodium azide and incubated at $37^{\circ} \mathrm{C}$ for $24 \mathrm{~h}$. Several colonies were picked and plated on MacConkey agar at $37^{\circ} \mathrm{C}$ for $24 \mathrm{~h}$. Transconjugants from MacConkey agar were subcultured on TSA, incubated at $37^{\circ} \mathrm{C}$ for $24 \mathrm{~h}$, and subjected to PCR for the optrA, poxtA, cfr, and fexA genes, respectively, according to Table 6 .

\subsection{Complete Genome Sequencing}

4.6.1. Genome Sequencing, Assembly, and Annotation

Total genomic DNAs from three strains were extracted using the DNeasy Blood and Tissue kit (Qiagen, Hilden, Germany) following the manufacturer's instructions. Libraries were prepared using the SMRTbell Express Template Prep kit 2.0 (Pacific Biosciences, Menlo Park, CA, USA) and subsequently sequenced using PacBio Sequel IIe (Pacific Biosciences). Raw reads were processed using demultiplex barcodes protocol, which is part of SMRT Link version 10.1, to obtain clean reads. Clean data were assembled using the microbial assembly protocol in the SMRT Link software. Genome annotations were performed using Rapid Annotation using Subsystem Technology server version 2.0 with default parameters [33]. The complete genome sequences of E. faecalis EFS17, EFS36, and EFS108 were deposited in GenBank under the accession numbers SUB10526591, SUB10526592, and SUB10526593, respectively.

\subsubsection{Bioinformatics Analysis}

The plasmid was identified using PlasmidFinder version 2.0 [34]. The identification of antimicrobial resistance genes in three genomes was performed using ResFinder version 4.1 [35]. ResFinder was set with default parameters, 90\% identity threshold, and $60 \%$ minimum length threshold. The circular plasmid maps of pEFS36_2 and pEFS108_1 were generated using SnapGene Viewer (https:/ / www.snapgene.com/snapgene-viewer/, accessed on 10 September 2021).

Supplementary Materials: The following are available online at https://www.mdpi.com/article/ $10.3390 /$ ijms222111335/s1, Table S1: Location of antimicrobial resistance genes identified in the three E. faecalis genomes.

Author Contributions: Conceptualization, H.-S.K. and H.-Y.K.; methodology, S.-W.S. and H.-S.K.; validation, M.-H.C. and G.-J.W.; investigation, S.-W.S. and Y.-S.G.; data curation, E.K. and S.-M.Y.; writing—original draft preparation, E.K., S.-W.S. and H.-S.K.; writing—review and editing, H.-Y.K.; visualization, E.K.; supervision, H.-Y.K.; project administration, H.-Y.K. All authors have read and agreed to the published version of the manuscript.

Funding: This research received no external funding. 
Institutional Review Board Statement: Not applicable.

Informed Consent Statement: Not applicable.

Data Availability Statement: The data presented in this study are available on request from the corresponding author.

Conflicts of Interest: The authors declare no conflict of interest.

\section{References}

1. Na, S.H.; Moon, D.C.; Choi, M.J.; Oh, S.J.; Jung, D.Y.; Kang, H.Y.; Hyun, B.H.; Lim, S.K. Detection of oxazolidinone and phenicol resistant enterococcal isolates from duck feces and carcasses. Int. J. Food Microbiol. 2019, 293, 53-59. [CrossRef] [PubMed]

2. Ma, X.; Zhang, F.; Bai, B.; Lin, Z.; Xu, G.; Chen, Z.; Sun, X.; Zheng, J.; Deng, Q.; Yu, Z. Linezolid Resistance in Enterococcus faecalis Associated With Urinary Tract Infections of Patients in a Tertiary Hospitals in China: Resistance Mechanisms, Virulence, and Risk Factors. Front. Public Health 2021, 9, 50. [CrossRef] [PubMed]

3. Tamang, M.D.; Moon, D.C.; Kim, S.R.; Kang, H.Y.; Lee, K.; Nam, H.M.; Jang, G.C.; Lee, H.S.; Jung, S.C.; Lim, S.K. Detection of novel oxazolidinone and phenicol resistance gene optrA in enterococcal isolates from food animals and animal carcasses. Vet. Microbiol. 2017, 201, 252-256. [CrossRef]

4. Bi, R.; Qin, T.; Fan, W.; Ma, P.; Gu, B. The emerging problem of linezolid-resistant enterococci. J. Glob. Antimicrob. Resist. 2018, 13, 11-19. [CrossRef]

5. Bender, J.K.; Fleige, C.; Klare, I.; Werner, G. Development of a multiplex-PCR to simultaneously detect acquired linezolid resistance genes cfr, optrA and poxtA in enterococci of clinical origin. J. Microbiol. Methods 2019, 160, 101-103. [CrossRef]

6. Yang, X.X.; Tian, T.T.; Qiao, W.; Tian, Z.; Yang, M.; Zhang, Y.; Li, J.Y. Prevalence and characterization of oxazolidinone and phenicol cross-resistance gene optrA in enterococci obtained from anaerobic digestion systems treating swine manure. Environ. Pollut. 2020, 267, 115540. [CrossRef]

7. Hua, R.; Xia, Y.; Wu, W.; Yang, M.; Yan, J. Molecular epidemiology and mechanisms of 43 low-level linezolid-resistant enterococcus faecalis strains in Chongqing, China. Ann. Lab. Med. 2018, 39, 36-42. [CrossRef]

8. Jung, Y.H.; Cha, M.H.; Woo, G.J.; Chi, Y.M. Characterization of oxazolidinone and phenicol resistance genes in non-clinical enterococcal isolates from Korea. J. Glob. Antimicrob. Resist. 2021, 24, 363-369. [CrossRef]

9. Egan, S.A.; Shore, A.C.; O'Connell, B.; Brennan, G.I.; Coleman, D.C. Linezolid resistance in Enterococcus faecium and Enterococcus faecalis from hospitalized patients in Ireland: High prevalence of the MDR genes optrA and poxtA in isolates with diverse genetic backgrounds. J. Antimicrob. Chemother. 2020, 75, 1704-1711. [CrossRef] [PubMed]

10. Hao, W.; Shan, X.; Li, D.; Schwarz, S.; Zhang, S.M.; Li, X.S.; Du, X.D. Analysis of a poxtA- And optrA-co-carrying conjugative multiresistance plasmid from Enterococcus faecalis. J. Antimicrob. Chemother. 2019, 74, 1771-1775. [CrossRef] [PubMed]

11. Park, K.; Jeong, Y.S.; Chang, J.; Sung, H.; Kim, M.N. Emergence of optrA-mediated linezolid-nonsusceptible enterococcus faecalis in a tertiary care hospital. Ann. Lab. Med. 2020, 40, 322-325. [CrossRef] [PubMed]

12. National Institute of Food and Drug Safety Evaluation; Ministry of Agriculture, Food and Rural Affairs; Animal and Plant Quarantine Agency; Korea Animal Health Products Association. National Antibiotic Use and Resistance Monitoring in 2019. 2020. Available online: https: / /impfood.mfds.go.kr/CFBCC02F02/getCntntsDetail?cntntsSn=325548\&cntntsMngId=00006 (accessed on 17 October 2021).

13. Kim, M.H.; Moon, D.C.; Kim, S.J.; Mechesso, A.F.; Song, H.J.; Kang, H.Y.; Choi, J.H.; Yoon, S.S.; Lim, S.K. Nationwide surveillance on antimicrobial resistance profiles of enterococcus faecium and enterococcus faecalis isolated from healthy food animals in South Korea, 2010 to 2019. Microorganisms 2021, 9, 925. [CrossRef]

14. Yoon, S.; Son, S.H.; Kim, Y.B.; Seo, K.W.; Lee, Y.J. Molecular characteristics of optrA-carrying Enterococcus faecalis from chicken meat in South Korea. Poult. Sci. 2020, 99, 6990-6996. [CrossRef] [PubMed]

15. Bae, S.H.; Yoon, S.; Kim, K.; Kim, Y.B.; Lee, Y.J. Comparative analysis of chloramphenicol-resistant enterococcus faecalis isolated from dairy companies in korea. Vet. Sci. 2021, 8, 143. [CrossRef]

16. Kang, Z.Z.; Lei, C.W.; Kong, L.H.; Wang, Y.L.; Ye, X.L.; Ma, B.H.; Wang, X.C.; Li, C.; Zhang, Y.; Wang, H.N. Detection of transferable oxazolidinone resistance determinants in Enterococcus faecalis and Enterococcus faecium of swine origin in Sichuan Province, China. J. Glob. Antimicrob. Resist. 2019, 19, 333-337. [CrossRef] [PubMed]

17. Maasjost, J.; Mühldorfer, K.; De Jäckel, S.C.; Hafez, H.M. Antimicrobial susceptibility patterns of enterococcus faecalis and enterococcus faecium isolated from poultry flocks in Germany. Avian Dis. 2015, 59, 143-148. [CrossRef] [PubMed]

18. Na, S.H.; Moon, D.C.; Kim, M.H.; Kang, H.Y.; Kim, S.J.; Choi, J.H.; Mechesso, A.F.; Yoon, S.S.; Lim, S.K. Detection of the phenicol-oxazolidinone resistance gene poxta in enterococcus faecium and enterococcus faecalis from food-producing animals during 2008-2018 in Korea. Microorganisms 2020, 8, 1839. [CrossRef]

19. Freitas, A.R.; Tedim, A.P.; Novais, C.; Lanza, V.F.; Peixe, L. Comparative genomics of global optrA-carrying Enterococcus faecalis uncovers a common chromosomal hotspot for optrA acquisition within a diversity of core and accessory genomes. Microb. Genom. 2020, 6, e000350. [CrossRef]

20. de Jong, A.; Simjee, S.; El Garch, F.; Moyaert, H.; Rose, M.; Youala, M.; Dry, M. Antimicrobial susceptibility of enterococci recovered from healthy cattle, pigs and chickens in nine EU countries (EASSA Study) to critically important antibiotics. Vet. Microbiol. 2018, 216, 168-175. [CrossRef] 
21. Wang, Y.; Lv, Y.; Cai, J.; Schwarz, S.; Cui, L.; Hu, Z.; Zhang, R.; Li, J.; Zhao, Q.; He, T.; et al. A novel gene, optrA, that confers transferable resistance to oxazolidinones and phenicols and its presence in Enterococcus faecalis and Enterococcus faecium of human and animal origin. J. Antimicrob. Chemother. 2015, 70, 2182-2190. [CrossRef] [PubMed]

22. He, T.; Shen, Y.; Schwarz, S.; Cai, J.; Lv, Y.; Li, J.; Feßler, A.T.; Zhang, R.; Wu, C.; Shen, J.; et al. Genetic environment of the transferable oxazolidinone/phenicol resistance gene optrA in Enterococcus faecalis isolates of human and animal origin. $J$. Antimicrob. Chemother. 2016, 71, 1466-1473. [CrossRef]

23. Chen, H.; Wang, X.; Yin, Y.; Li, S.; Zhang, Y.; Wang, Q.; Wang, H. Molecular characteristics of oxazolidinone resistance in enterococci from a multicenter study in China. BMC Microbiol. 2019, 19, 162. [CrossRef]

24. Kehrenberg, C.; Schwarz, S. Florfenicol-chloramphenicol exporter gene fexA is part of the novel transposon Tn558. Antimicrob. Agents Chemother. 2005, 49, 813-815. [CrossRef]

25. Shan, X.; Li, X.S.; Wang, N.; Schwarz, S.; Zhang, S.M.; Li, D.; Du, X.D. Studies on the role of IS1216E in the formation and dissemination of poxtA-carrying plasmids in an Enterococcus faecium clade A1 isolate. J. Antimicrob. Chemother. 2020, 75, 3126-3130. [CrossRef]

26. Darini, A.L.C.; Palepou, M.F.I.; Woodford, N. Effects of the movement of insertion sequences on the structure of vana glycopeptide resistance elements in Enterococcus faecium. Antimicrob. Agents Chemother. 2000, 44, 1362-1364. [CrossRef]

27. Raze, D.; Dardenne, O.; Hallut, S.; Martinez-Bueno, M.; Coyette, J.; Ghuysen, J.M. The gene encoding the low-affinity penicillinbinding protein $3 \mathrm{r}$ in Enterococcus hirae S185R is borne on a plasmid carrying other antibiotic resistance determinants. Antimicrob. Agents Chemother. 1998, 42, 534-539. [CrossRef]

28. Ciric, L.; Brouwer, M.S.M.; Mullany, P.; Roberts, A.P. Minocycline resistance in an oral Streptococcus infantis isolate is encoded by tet(S) on a novel small, low copy number plasmid. FEMS Microbiol. Lett. 2014, 353, 106-115. [CrossRef] [PubMed]

29. Clinical and Laboratory Standards Institute. Performance Standards for Antimicrobial Susceptibility Testing; CLSI: Wayne, PA, USA, 2014; Volume 32, ISBN 1562388975.

30. Ruiz-Garbajosa, P.; Bonten, M.J.M.; Robinson, D.A.; Top, J.; Nallapareddy, S.R.; Torres, C.; Coque, T.M.; Cantón, R.; Baquero, F.; Murray, B.E.; et al. Multilocus sequence typing scheme for Enterococcus faecalis reveals hospital-adapted genetic complexes in a background of high rates of recombination. J. Clin. Microbiol. 2006, 44, 2220-2228. [CrossRef] [PubMed]

31. Francisco, A.P.; Vaz, C.; Monteiro, P.T.; Melo-Cristino, J.; Ramirez, M.; Carriço, J.A. PHYLOViZ: Phylogenetic inference and data visualization for sequence based typing methods. BMC Bioinform. 2012, 13, 87. [CrossRef] [PubMed]

32. Aljahdali, N.H.; Khajanchi, B.K.; Weston, K.; Deck, J.; Cox, J.; Singh, R.; Gilbert, J.; Sanad, Y.M.; Han, J.; Nayak, R.; et al. Genotypic and phenotypic characterization of incompatibility group fib positive salmonella enterica serovar typhimurium isolates from food animal sources. Genes 2020, 11, 1307. [CrossRef]

33. Aziz, R.K.; Bartels, D.; Best, A.; DeJongh, M.; Disz, T.; Edwards, R.A.; Formsma, K.; Gerdes, S.; Glass, E.M.; Kubal, M.; et al. The RAST Server: Rapid annotations using subsystems technology. BMC Genom. 2008, 9, 75. [CrossRef] [PubMed]

34. Carattoli, A.; Hasman, H. PlasmidFinder and In Silico pMLST: Identification and Typing of Plasmid Replicons in Whole-Genome Sequencing (WGS). In Methods in Molecular Biology; H. Humana: New York, NY, USA, 2020; Volume 2075, pp. $285-294$.

35. Bortolaia, V.; Kaas, R.S.; Ruppe, E.; Roberts, M.C.; Schwarz, S.; Cattoir, V.; Philippon, A.; Allesoe, R.L.; Rebelo, A.R.; Florensa, A.F.; et al. ResFinder 4.0 for predictions of phenotypes from genotypes. J. Antimicrob. Chemother. 2020, 75, 3491-3500. [CrossRef] 